\title{
SALT as an African Facility
}

\author{
Peter Martinez
}

South African Astronomical Observatory, P.O. Box 9, Observatory 7935 South Africa. e-mail: peter@saao.ac.za

\begin{abstract}
Over the next five years, South Africa and its international partners will construct the Southern African Large Telescope (SALT), a. 10-m optical/near-infrared spectroscopic survey telescope. This paper traces the arguments used to justify the construction of such a facility in Africa. The collateral benefits expected from this project are discussed, along with suggestions of how to realize these benefits. SALT has the potential to promote the development of astronomy on the continent. The creation of an African Network for Astronomy Education and Research is proposed as a way to stimulate such development.
\end{abstract}

\section{A Large African Telescope}

Since the early 1970 s the major facility for optical/infrared astronomy in subSaharan Africa has been the South African Astronomical Observatory (SAAO) in Sutherland. This facility has made many major contributions to southern hemisphere astronomy. The largest of the SAAO telescopes is the $1.9-\mathrm{m}$ Radcliffe reflector. By exploiting advances in detector quantum-efficiency and in computing power, this telescope has remained a significant facility for modern southern hemisphere astronomy. However, by the $1980 \mathrm{~s}$ it became clear that South African astronomers needed a successor to the aging 1.9-m telescope at Sutherland.

In the early $1990 \mathrm{~s}$ a case was made to construct a $3.5-\mathrm{m}$ telescope similar to ESO's New Technology Telescope. This was a modest aspiration, coming at a time when other countries were planning to build $8-\mathrm{m}$ to $10-\mathrm{m}$ class telescopes, but it seemed to be the best that South Africa could afford. This case for a large national telescope was being made in parallel with the great political changes taking place in South Africa at the time. The right political context for a large national telescope project did not exist at the time, and it remained a notional project.

Then, in 1994, a remarkable thing happened; South Africa experienced a peaceful transition to a democratically elected, majority government. One of the processes that the new government set in train was an audit of South Africa's science and technology capability. The resulting policy document concluded that "scientific endeavour is not purely utilitarian in its objectives and has important associated cultural and social values ... Not to offer 'flagship' sciences (such as physics and astronomy) would be to take a negative view of our future the view that we are a second-class nation, chained forever to the treadmill of 


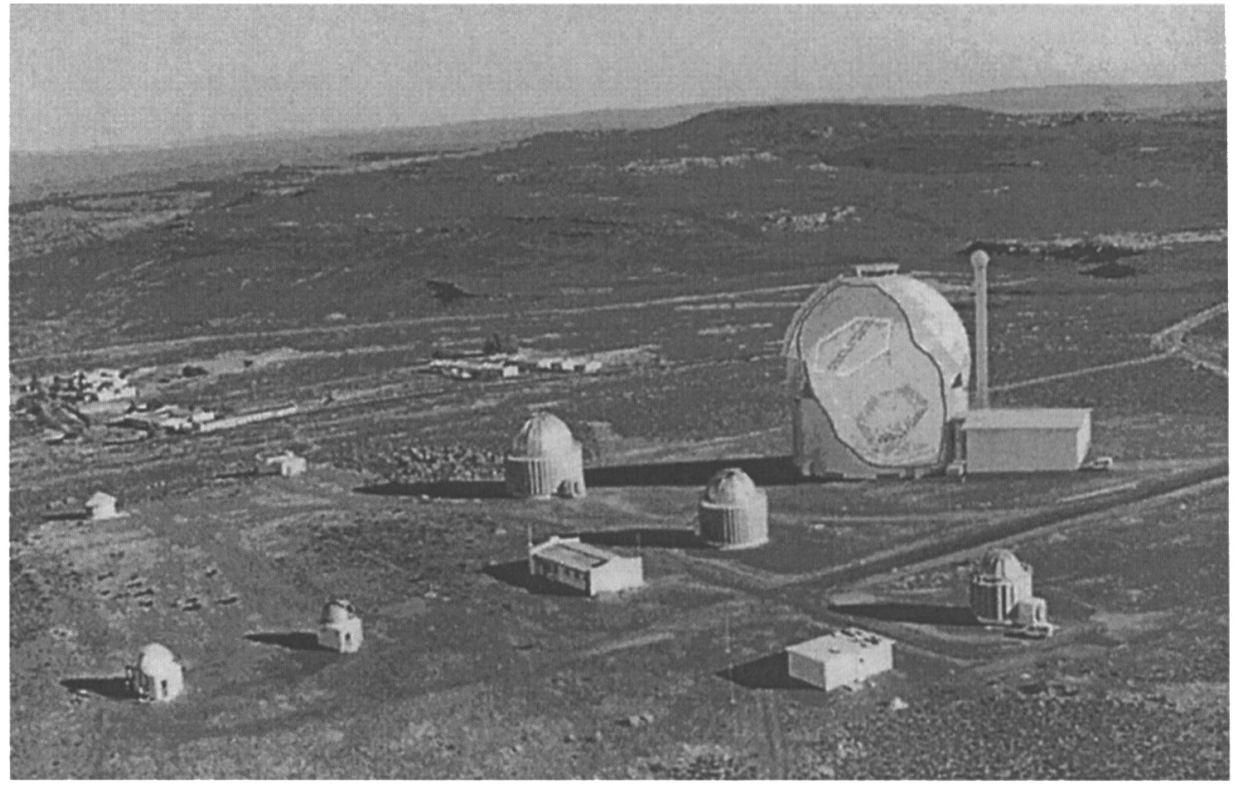

Figure 1. Artist's conception of SALT as it will appear among the existing telescopes in Sutherland.

feeding and clothing ourselves." In other words, this newly elected government had recognized the value of science and technology as an agent for national transformation.

Concurrently with these political developments, but half a world away, a consortium of American and German astronomers was constructing the HobbyEberly Telescope (HET), in Texas. This telescope represents a radical departure from conventional telescope design, and offers much of the functionality of a conventional telescope at a fraction of the cost. By the mid-1990s, the builders of the HET were thinking of a southern-hemisphere twin of their telescope, and they approached their South-African colleagues, with whom they already had strong ties. The idea was enthusiastically adopted by South-African astronomers as this offered the possibility of building a 10-m class telescope for a price similar to that of the more modest $3.5-\mathrm{m}$ telescope project, which seemed to be going nowhere. Moreover, with SAAO's well-developed infrastructure in Sutherland, it seemed likely that South Africa would succeed in attracting international partners to join a $10-\mathrm{m}$ telescope project in the southern hemisphere. The project soon attracted the interest and support of the country's science administration, and lobbying for the Southern African Large Telescope (SALT) at top government level began in earnest in 1997.

On 1 June 1998 the South-African Minister of Arts, Culture, Science and Technology announced in Parliament that the South African Cabinet had approved the construction of SALT on condition that the South African Government would fund $50 \%$ of the cost and the balance would have to be funded by international partners. There was unanimous support from all political parties for this announcement. By November 1999, the Minister was satisfied that suff- 


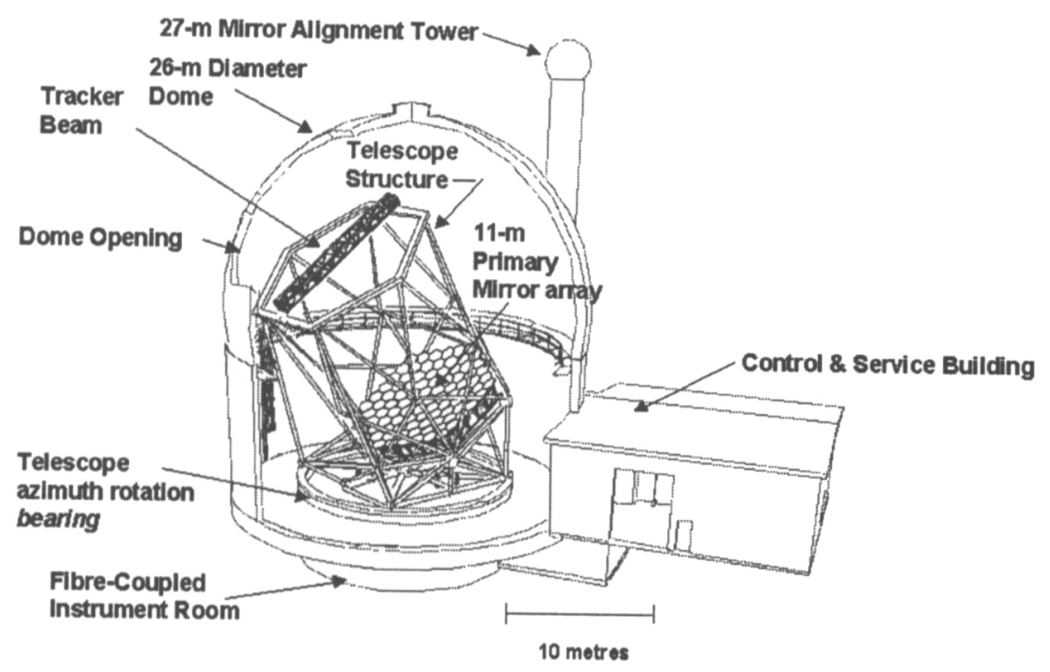

Figure 2. Schematic view of the SALT facility. (Image courtesy of the HET Board.)

cient international funding had been committed to authorize the commencement of expenditure of the South African funds allocated for this project.

As of August 2000, the SALT partnership included South Africa, the HET Board, Poland, Rutgers University, Göttingen University, the University of Wisconsin-Madison, Carnegie Mellon University, Canterbury University (New Zealand) and a consortium of U.K. universities comprising the University of Central Lancashire, Keele University, Nottingham University and Southampton University, and Armagh Observatory in Northern Ireland. As of this writing, SALT is about $90 \%$ funded for construction and the first 10 years of operations. Prospective additional partners are in the process of raising funds to join SALT.

How does South Africa justify building one of the world's largest telescopes when it has so many other pressing needs? This paper outlines the ideas that have emerged (and continue to emerge) in response to this question. Section 2 contains a brief description of the design of SALT. Section 3 discusses current plans for a programme of collateral benefits to be implemented during the construction and operation phases of SALT. Section 4 discusses how to promote the use of SALT as an African facility.

\section{The Design of SALT}

SALT is based on the design of the Hobby-Eberly Telescope (HET), which was built at McDonald Observatory, in Texas. For a description of the HET project, refer to the paper by Ramsey et al. (1998) and references therein. Briefly, the HET design concept is as follows. The telescope has a segmented spherical primary mirror array 11 metres across. The mirror array comprises 91 hexagonal mirrors, all 1 metre across. This mirror array is supported in an open framework 
structure inclined at a fixed tilt ( 35 degrees, in the case of SALT). The telescope structure moves only in azimuth (not elevation), and only when slewing. Rotation in azimuth is accomplished by means of floating the telescope structure on an air cushion. During an observation, the telescope structure remains stationary, and tracking motions are accomplished by a tracker beam that moves across the top hexagon of the telescope structure.

In one full rotation, the telescope sweeps out a $12^{\circ}$-wide annulus on the sky, centred on the telescope's latitude. Astronomical objects are accessible to this telescope only when passing through this 'annulus of visibility'. The amount of time it takes an object to cross the annulus of visibility is declination-dependent. At the worst extreme, near the celestial equator, objects can be tracked for about 48 minutes, while at the southern extreme of the annulus of visibility, objects can be tracked for up to 2.4 hours. This feature of the telescope's operation dictates that observations are done in a queue-scheduled mode. That is, unlike a conventional telescope, which is dedicated to one programme for a whole night, SALT will typically observe targets from many different programmes during the same night.

The fixed tilt of the telescope simplifies the structure considerably since the gravity load on all structural members and the mirror array is constant. Likewise, the use of a spherical primary means that all 91 mirror segments are identically figured. Together, these cost-saving features allow access to $70 \%$ of the sky accessible to a fully steerable conventional telescope at $20 \%$ of the cost.

SALT will operate in the wavelength region of 0.3 to 2.5 microns. The thermal background increases rapidly redward of 2.5 microns, and the telescope is not efficient at those longer wavelengths. Moreover, the altitude of Sutherland $(1757 \mathrm{~m})$ makes it uncompetitive in the thermal infrared, compared to other high-altitude sites.

One of SALT's most complex subsystems is the tracker beam, which moves across the top hexagon of the telescope structure to accomplish tracking. This tracker beam weighs 4.5 tons and must be capable of 9 degrees of freedom to move accurately on a spherical focal surface with a linear precision of 6 microns. The tracker beam will support the Prime Focus Instrument Platform (PFIP), a package housing the spherical-aberration corrector, atmospheric-dispersion corrector, exit-pupil baffle system, acquisition/guide CCD camera, a Prime Focus Imaging Spectrograph (PFIS), and a fibre feed to medium-resolution and highresolution spectrographs in an environmentally controlled room under the telescope. For a more detailed discussion of SALT instrumentation plans, refer to the paper by Buckley et al. (2000).

SALT is a copy of the HET, but not an identical copy. The HET Board is a partner in the SALT project, and the SALT project team is benefiting from the hindsight of the team that built the HET. The tilt of SALT has been changed from HET's $35^{\circ}$ tilt to $37^{\circ}$ to allow SALT to access most of the Small Magellanic Cloud. SALT will also incorporate edge-sensors to facilitate alignment of the primary mirror array, a modification soon to be applied to the HET as well. The most significant departure from the HET design is in the manner in which spherical aberration is corrected in SALT. A new design by O'Donoghue (2000) promises to deliver substantially better-quality images, an 8-arcmin field (double that of the HET), somewhat reduced vignetting, and an increased pupil size 
(pending implications of redesigning the top hexagon). The differences between SALT and HET are discussed in more detail by Stobie et al. (2000).

\section{The SALT Collateral Benefits Plan}

The decision to build SALT was taken at the highest levels in South Africa. By funding SALT, the South-African government is making the statement that it sees South Africa as a significant role player in the international scientific community. SALT will enable South-African scientists to remain internationally competitive in astronomy well into the 21st century. However, SALT has an importance to South Africa (and Africa) far beyond its astronomical research mission. SALT will provide distinct and substantial benefits in the development of people, technology, and the economy. The SALT Collateral Benefits Plan is designed to maximize the benefits from the investment of public funds in the construction and operation of SALT. There are three main thrusts to this plan: industrial empowerment, educational empowerment, and public outreach. The Collateral Benefits Plan is being drafted in consultation with stakeholders from government, industry and education. The discussion in the remainder of this section draws heavily on the discussion document 'SALT Collateral Benefits Plan' (1999).

\subsection{SALT industrial empowerment}

The SALT project is being planned in such a manner as to maximize the industrial pay-offs from the development of components, subsystems, equipment, and labour for the construction and provision of SALT. Specific elements include a procurement policy with emphasis on empowerment and capacity building of people, firms and companies from previously disadvantaged communities. Foreign companies contracted to work on SALT could host South-African technical personnel at the contractor's facility to collaborate in the design and manufacturing efforts. Careful selection of personnel will maximise this benefit.

The result of these activities will be industrial collaborations joining South African and foreign companies in new ways to increase technical capabilities. This will result in technology transfer in the areas of optics, electronics, micropositioning, dynamic control systems, precision sensing, and others. These companies will be able to compete for additional business within and outside of South Africa.

\subsection{SALT educational empowerment}

One of the biggest educational challenges confronting South Africa is to broaden the science, education and technology training base, particularly in the black community. SALT can play a significant role in meeting this challenge by providing educational and training opportunities for astronomers, physicists, computer scientists, and engineers during the construction ( $5 \mathrm{yr}$ ) and operational $(25+\mathrm{yr})$ phases of the project. This will specifically involve student participation in the SALT project team, student placement with SALT contractors, student participation in collaborations with SALT partners, and student involvement in instrumentation development. Since these students all have to be registered at 
their own academic institutions, this will promote closer ties between SAAO and those institutions, and empower them in new technologies.

The involvement of students in all phases of the SALT project will equip them with the experience of participation in a large, complex, high-technology project, which experience will be carried into later careers. Knowledge of the technology, interactions and techniques for development of such projects is vital, as South Africa must address ever more ambitious technical undertakings. Experience with SALT will provide confidence in using SALT's constituent technologies in future projects.

Another component of this educational thrust is to promote the development of astronomy at the historically black universities in South Africa, where the subject has not featured in the past. This will have the advantage of increasing the numbers of scientists and engineers participating in the construction and use of SALT, and will also make SALT more accessible to the wider community in South Africa. The achievement of research success in astronomy at these institutions will have long-term spin-offs in terms of encouraging success in other fields at those universities by stimulating both students and faculty. The SAAO will play a key role in implementing these policies.

The astronomical activities generated by the SALT project will provide excellent training opportunities in astronomy. However, the nature of the training is such that students will be well equipped to pursue challenging careers in other disciplines should they wish to leave astronomy. There is a growing trend internationally for students trained in astronomy and astrophysics (particularly at post-graduate level) to be sought after for employment in business and industry for their analytic and problem-solving skills.

\subsection{Public outreach and direct education benefits}

The South-African government recognizes that scientific literacy is the key to ensuring the technological competitiveness of the nation. Until recently, black people in South Africa had very limited access to science, engineering and technology education, training and careers, with the result that the majority of the labour pool is unskilled in this vital area. South Africa requires a sustained campaign to promote awareness and understanding of science, engineering and technology at all levels in society. SALT will contribute to this process by stimulating excitement about science and technology in general.

A careful programme of educational and public outreach benefits is being compiled in collaboration with other stakeholders to capitalize on the interest generated by SALT. This programme includes activities such as training of educators in astronomy, a 'Starbus' science roadshow for outreach to rural communities, development of educational resources, etc. To accommodate the large numbers of visitors expected to visit SALT, a Visitor Centre will be constructed in Sutherland. The Visitor Centre will contain interactive hands-on displays on SALT, astronomy, and other scientific aspects related to the Sutherland/Karoo region. For operational reasons the Visitor Centre will be in a separate building from SALT, but the SALT telescope dome will incorporate a special viewing gallery where visitors will be able to witness the full scale of the telescope at close quarters. This facility is discussed in detail by Rijsdijk (2001) elsewhere in this volume. 


\section{SALT as an African facility}

Capability in the space sciences will be a vital factor in the development of Africa in the 21st century. The applied space sciences are underpinned by the basic space sciences, such as astronomy and astrophysics. One of the indicators of activity in basic space science is membership of the International Astronomical Union (IAU). This indicator reveals that Africa is very poorly represented in the space sciences, compared to other regions in the world. Currently, only four out of 53 African states are members of the IAU. As of 1999, the total IAU membership among African countries amounted to 95 persons, or $1.1 \%$ of the total IAU membership. The African membership of the IAU is dominated by South Africa (48\%) and Egypt (41\%), followed by Nigeria, Algeria, Morocco and Mauritius, each with $4 \%$ or fewer members. Not surprisingly, these countries are among the more prosperous nations on the continent.

One should bear in mind that the above statistics reflect the presence of nationally organized astronomical communities. There are many individual scientists distributed throughout Africa who are involved in astronomy education, and in some cases research also, who are not members of the IAU. Isolation, and lack of access to world-class facilities, are the two factors that retard the development of basic space science in Africa.

At present there is a dearth of large-scale astronomical facilities in Africa, and African astronomers are forced to develop their careers elsewhere. The creation of new large-scale facilities like SALT will contribute to encouraging highly trained professionals to remain in their own cultural and political environment. As the development agenda of the region would likely preclude the construction of another facility comparable to SALT in the foreseeable future, this project was conceptualised from the start as one that would serve the needs of the region. SALT has been intentionally named the Southern African Large Telescope, as opposed to the South-African Large Telescope, to underscore the fact that this is seen as a regional project. However, if there is to be an African user community for SALT by the time that the telescope is commissioned in 2005, then the first steps have to be taken now to develop that community.

In order to promote the development of astronomy in Africa, one has to provide adequate opportunities for African astronomers to practice their science in Africa, rather than pursuing careers elsewhere. Networking is the key to establishing a critical mass of African astronomers working in Africa. Networking breaks the isolation of scientists by involving them in a distributed community of scientists working towards a common goal in the same field, thereby giving them access to the collective expertise and experience of that whole group.

The advent of the Internet is revolutionizing science by giving scientists everywhere unprecedented access to information and data. Although African scientists may not enjoy the same standards of connectivity as their European or North-American colleagues, many now have access to electronic mail, and limited access to the worldwide web. This access provides the foundation upon which to build an African Network for Education and Research in Astronomy, whose hub could be the optical and radio observatories in South Africa. Such a network would allow astronomers from a variety of African countries to access instruments at one the best astronomical sites on the continent, without crippling investments in their own countries. 
The Working Group on Space Sciences in Africa (Martinez 2000) and the South-African Astronomical Observatory are currently planning a pilot project which could form the basis of an African Network for Education and Research in Astronomy. This is to be implemented through a Fellowship programme, hosted by SAAO. Fellows from different African countries would work together at SAAO for a period of several months, during which time they would develop research skills in astronomy, as well as the personal acquaintances so important in scientific collaboration. At the same time they would collaborate on the development of educational resources which could be used upon their return to their home institutions.

The programme for the Fellowship should be devised in such a way as to ensure the long-term goal of establishing a sustainable network after Fellows return to their home institutions. The scientific programme must be developed in such a manner that it is topical, yet accessible to physicists who may have little or no background in astrophysical techniques. The networked approach demands that the participants should work on a common project, or 'key theme'. The key theme should be chosen with regard to scientific relevance, modest computing hardware and software requirements, and modest data storage and data transfer requirements. In time, the network will reach a critical mass, after which it may be capable of addressing other key themes.

It is important to structure the network in such a way that scientists may fruitfully collaborate with each other from their home institutions. This will be accomplished through providing Fellows with a common set of software tools and teaching resources. Some measure of remote access (via email) to a robotic telescope at SAAO could also be included. In this way the participating scientists could continue to obtain new data of excellent quality for their own research projects.

If this pilot project is successful, a new group of Fellows will be selected each year, and the network will grow with time. In this way, there could be a number of potential users of SALT by the time the telescope is commissioned in 2005.

Acknowledgments. The author gratefully acknowledges financial support from the IAU and the South African Astronomical Observatory for participation in this Special Session.

\section{References}

Buckley, D.A.H., O’Donoghue, D.O., Sessions, N.J., Nordsieck, K.H. 2000, Instrumentation options for the Southern African Large Telescope, Proc. SPIE, in press

Martinez, P. 2000, Bulletin on Teaching of Astronomy in the Asia-Pacific Region, (See also http://www.saao.ac.za/ wgssa)

O'Donoghue, D.O. 2000, The correction of spherical aberration in the Southern African Large Telescope (SALT), Proc. SPIE, in press

Ramsey, L.W., et al. 1998, The early performance and status of the Hobby-Eberly telescope, in Advanced Technology Optical/IR Telescopes VI, Proc. SPIE, $3352,34-42$ 
Rijsdijk, C. 2001, this volume, pp. 117-130

SALT Collateral Benefits Plan 1999, South African Astronomical Observatory/National Research Foundation

Stobie, R.S., Meiring, K., Buckley, D.A.H. 2000, Design of the Southern African Large Telescope, Proc. SPIE, in press

Discussion Batten noted that Martinez had mentioned five African countries are members of the IAU. At this Session we had representatives from seven African countries - probably a record for the IAU and a tribute to the energy that Martinez had put into the UN-sponsored Working Group on Space Sciences in Africa. Hemenway asked if the pilot fellowship scheme described by Martinez was confined to university level, or whether it was also open to primary-school and secondary-school teachers. Martinez replied that it does not at present include school teachers but thanked her for the suggestion. 